\title{
Estimación del área y el número máximo de turistas para observar la anidación de tortugas marinas protegidas
}

\author{
Nelson Espinoza Mora ; Carlos M. Orrego ${ }^{2} \&$ Luis D. Alfaro Alvarado ${ }^{3,}$, \\ ${ }^{1}$ Posgrado en Ciencias Marinas y Costeras, Universidad Nacional, Costa Rica. ${ }^{2}$ Ministerio de Ambiente y Energía, Costa Rica. \\ ${ }^{3}$ Instituto Internacional en Conservación y Manejo de Vida Silvestre, Universidad Nacional, Costa Rica.
}

\begin{abstract}
Resumen. Las áreas protegidas tienden a resguardar ecosistemas poco intervenidos y constituyen un foco de alta atracción para el turismo. Por ambas razones resulta clave regular su visitación a través de herramientas de gestión de visitas. En el presente trabajo se desarrolló una metodología para estimar el área de observación responsable de las tortugas marinas durante su proceso de anidación por parte de turistas en áreas protegidas marino-costeras, tomando como sitio de aplicación el Refugio Nacional de Vida Silvestre Camaronal. Esta metodología utiliza variables relacionadas con el comportamiento anidatorio de la tortuga lora (Lepidochelys olivacea) a fin de determinar el área y la cantidad de observadores recomendable bajo ciertas limitaciones de espacio y considerando los arribos de tortugas registrados durante el período 2013-2015. En esos tres años se registraron 15515 arribos de tortuga lora relacionados con las fases lunares; la mayor cantidad de arribos sucedió estando la luna en cuarto menguante. El área propuesta para observar a las tortugas marinas en el sitio Camaronal fue $51.4 \mathrm{~m}^{2}$ y el área de observación de una tortuga marina corresponde a un círculo de $~ 500$ $\mathrm{m}^{2}$ en grupos turísticos de 10 personas como máximo. El comportamiento de anidación no se vería alterado significativamente mientras se respete el tamaño de grupo sugerido. El refugio tiene como uno de sus objetivos de conservación a L. olivacea, por lo cual la preservación de esta especie debe ser prioridad.
\end{abstract}

[Palabras clave: Lepicholeys olivacea, manejo de vida silvestre, ecología aplicada]

\begin{abstract}
Aвstract. Estimate of the maximum area and number of tourists observing turtle nesting in marine protected areas. Protected areas tend to preserve conserved ecosystems and constitute a focus of high attraction for tourism. For both reasons, it is essential to regulate their visitation through management tools. In this study, a methodology was developed to estimate the area of responsible observation of sea turtles by tourists during their nesting process in marine-coastal protected areas, using the Camaronal National Wildlife Refuge as an application site. This methodology uses variables related to olive ridley turtle (Lepidochelys olivacea) nesting behavior in order to determine the area and number of observers recommended under certain space limitations and considering the turtle arrivals recorded during the 2013-2015 period. In those three years, 15515 olive ridley turtle arrivals were recorded in relation to the lunar phases; the highest number of arrivals occurred during the waning quarter of the moon. The proposed area to observe sea turtles at the Camaronal site was $51.4 \mathrm{~m}^{2}$ and the area to observe a sea turtle corresponds to a circle of $\sim 500 \mathrm{~m}^{2}$ in tour groups of 10 people maximum. Respecting the suggested group size would not significantly alter nesting behavior. The refuge has $L$. olivacea as one of its conservation objectives, so the preservation of this species should be a priority.
\end{abstract}

[Palabras clave: Lepicholeys olivacea, wildlife management, applied ecology]

\section{INTRODUCCIÓN}

Con el reconocimiento actual de la amplitud y la magnitud de las amenazas a la biodiversidad se ha vuelto necesario el manejo de poblaciones silvestres dentro y fuera de las áreas protegidas (Pullin et al. 2004; Dudley and Philips 2006; Chacon and Soley 2020). Las decisiones de manejo tienen un impacto directo sobre los objetos de conservación que se protegen o utilizan, y por esto se espera que sean tomadas basándose en evidencia técnica y rigurosa (Polasky et al. 2011; Chacon and Soley 2020). Sin embargo, la información necesaria para manejar los objetos de conservación a menudo es poca o inexistente, en especial cuando se habla de áreas marinas protegidas (Lundquist and Granek 2005; Chacon and

Editor asociado: Sergio Lambertucci

\laalfaro@una.ac.cr
Soley 2020). Las áreas protegidas tienden a resguardar ecosistemas poco intervenidos y su accesibilidad reducida genera un foco muy atractivo para el turismo (Garay et al. 2008). De ahí la importancia de regular su visitación por medio de herramientas de gestión.

Costa Rica cuenta con una gran diversidad de hábitats costeros (Cortés and Jiménez 2003). Dentro de esa diversidad se incluyen las especies de tortugas marinas, con un ámbito de distribución geográfica amplio (Camacho et al. 2008). El país cuenta con cinco especies de tortugas de las siete reportadas a nivel mundial, pertenecientes a dos grandes familias: Dermochilidae (cuyo único representante actual es la baula, Dermochelys 
coriacea) y Chelonidea (que contiene cuatro especies: la verde del pacífico o negra [Chelonia mydas], la lora [Lepidochelys olivácea], la tortuga carey [Eretmochelys imbricata] y la cabezona [Caretta caretta] [Bickhamet al. 2007]). Según la lista roja de la Unión Internacional para la Conservación de la Naturaleza (UICN), todas estas especies están amenazadas, excepto la tortuga lora, que se encuentra como vulnerable y en el Apéndice I de la Convención sobre el Comercio Internacional de Especies Amenazadas de Fauna y Flora Silvestres (CITES). En el Apéndice I de la Convención de Especies Migratorias (CMS) y en la legislación nacional se encuentran clasificadas en la lista de especies de fauna con poblaciones amenazadas o reducidas (Resolución 092-2017 del Reglamento Nro. 40548 de la Ley de Conservación de Vida Silvestre No 7317). Las poblaciones de estas especies se encuentran en estado crítico principalmente por causas antrópicas como la explotación de los recursos marinos y las interacciones negativas con tipos de artes o aparejos de pesca (e.g., redes de arrastre, redes agalleras, palangres pelágicos y de fondo) y desechos de artes de pesca lanzados al mar (Rosales et al. 2010). Otra causa antrópica que afecta a las poblaciones de las tortugas es la fragmentación de su hábitat en las áreas de alimentación y anidación. Esto se debe, sobre todo, al desarrollo costero y al incremento del turismo sin una debida planificación de ordenamiento espacial marino-costero, y también a la diversificación de actividades realizadas tanto en las zonas costeras como en las oceánicas (Gámez et al. 2009). En este último caso, el turismo representa una fuerte amenaza para el comportamiento de las tortugas marinas y de otras especies.

Se han documentado cambios en el comportamiento de las tortugas durante actividades turísticas como caminatas, buceo apnea y buceo autónomo (Labrada 2003). En términos generales, los animales se asustan e, incluso, huyen de los sitios habituales, con lo cual interrumpen o modifican su comportamiento natural. Por ejemplo, para la especie $C$. caretta se describieron diferentes comportamientos de anidación en la costa central de Florida, EE.UU., y se encontró que a mayor número de observadores se afectaba la anidación de la especie (Johnson et al. 1996). Los observadores pueden afectar a las tortugas tanto en sus actividades de alimentación como en sus faenas de limpieza, en el descanso y en el apareamiento (González and Cubero 2010).
En los últimos años, el turismo recreativo en áreas protegidas se convirtió en una actividad económica importante, ya que es un sector altamente productivo, sobre todo en Costa Rica (Brenes et al. 2004). Los planes de manejo de las áreas silvestres protegidas (ASP) son los instrumentos para planificar la gestión de las ASP. Para su creación, las ASP que tienen como objetivo de conservación a las tortugas marinas (como el Refugio Nacional de Vida Silvestre Camaronal) deben tener un plan específico para el turismo en el que se defina la capacidad de carga de la visitación, el reglamento de uso público, la zonificación, el número de visitantes con guía para el tour y los horarios de atención, entre otros aspectos. A través del Sistema Nacional de Áreas de Conservación (SINAC), el Ministerio de Ambiente y Energía (MINAE) realiza un monitoreo anual de la efectividad de la gestión de las ASP aplicando la guía validada para esta evaluación a nivel nacional. En este monitoreo se hacen recomendaciones de manejo adaptativo para mejorar la gestión del ASP en cuanto al turismo, que, por legislación nacional, es una de las actividades permitidas en las áreas silvestres protegidas. La medición de los impactos del turismo no se debe basar sólo en un número específico, sino en una gama de procesos que le permita a los administradores del área tomar decisiones de manejo y medir los resultados (Huaraca 2008).

La capacidad de acogida de turistas se puede estimar en base a la capacidad de soporte del suelo y a los servicios disponibles (Aranguren et al. 2008). En 1986 se propuso la metodología de capacidad de carga turística (CCT) como herramienta fundamental para gestionar el turismo en espacios de conservación a escala mundial (O'Reilly 1986). Esta metodología se caracteriza por tomar en cuenta la capacidad de manejo por parte del personal del ASP, de las instalaciones y de las condiciones climáticas, entre otros puntos. La CCT define el número de turistas que pueden permanecer en un ASP durante un período de tiempo determinado sin afectar de manera significativa ni la integridad de los ecosistemas protegidos ni, en determinadas APS, el legado cultural de edificaciones, estructuras, obras viales o acueductos, etc. (Cifuentes 1992). A su vez, existe otra metodología creada a mitad de la década del ochenta, pero que tomó auge en la gestión del turismo en ASP, conocida como el método de Limites de Cambio Aceptable (LCA), que se basa en la definición de estándares de calidad de un área 
natural e identifica qué cambios son aceptables para el uso adecuado de cada ASP (Stankey et al. 1985).

En el presente trabajo se realizó la estimación del área de observación requerida para no alterar el comportamiento de anidación de las tortugas marinas, tomando como objeto de estudio la especie L. olivacea en el Refugio Nacional de Vida Silvestre Camaronal (RNVSC). Para esto se determinaron variables de comportamiento de la especie, espaciales y relacionadas con el manejo de grupos de turistas.

\section{Materiales y Métodos}

\section{Sitio de estudio}

El RNVSC, ubicado en Guanacaste, en el Pacifico de Costa Rica, posee $2.10 \mathrm{~km}^{2}$ terrestres y $22.224 \mathrm{~km}^{2}$ de área marina, incluyendo los $200 \mathrm{~m}$ de la zona marítima terrestre (ZMT) desde Punta el Roble hasta la parte sur de Islita (Figura 1). Este refugio abarca ecosistemas de acantilados rocosos, bosques primarios y secundarios, manglares, esteros, pastizales arbolados y bosque rivereño asociado al río
Ora, sistemas pelágicos y bénticos y una playa arenosa de tres kilómetros. La playa, de 3000 m de longitud, es sitio de anidación para cuatro especies de tortugas marinas que visitan el Pacifico de Costa Rica: la baula, la verde o negra del pacifico y la lora. Sin embargo, L. olivácea es la más abundante y sobre la cual se concentran los mayores esfuerzos de conservación en el sitio (SINAC 2014). El RNVSC presenta un clima de la zona con temperaturas medias anuales de 24 a $27^{\circ} \mathrm{C}$ y la precipitación anual promedio entre 2013-2015 rondó los $2328 \mathrm{~mm}$, con un promedio anual de 125 días con lluvia y un período seco de seis meses (IMN 2015); el período se extiende desde mediados de noviembre hasta finales de abril (Rojas 2011). El RNVSC cuenta con un total de 60 mojones colocados y enumerados cada $50 \mathrm{~m}$ de norte a sur a lo largo de la playa arenosa (Figura 1).

\section{Gestión actual del turismo de observación de tortugas marinas}

La observación de tortugas marinas en el Refugio Nacional de Vida Silvestre Camaronal es una actividad propia de la zona producto de una demanda de influencia al refugio por

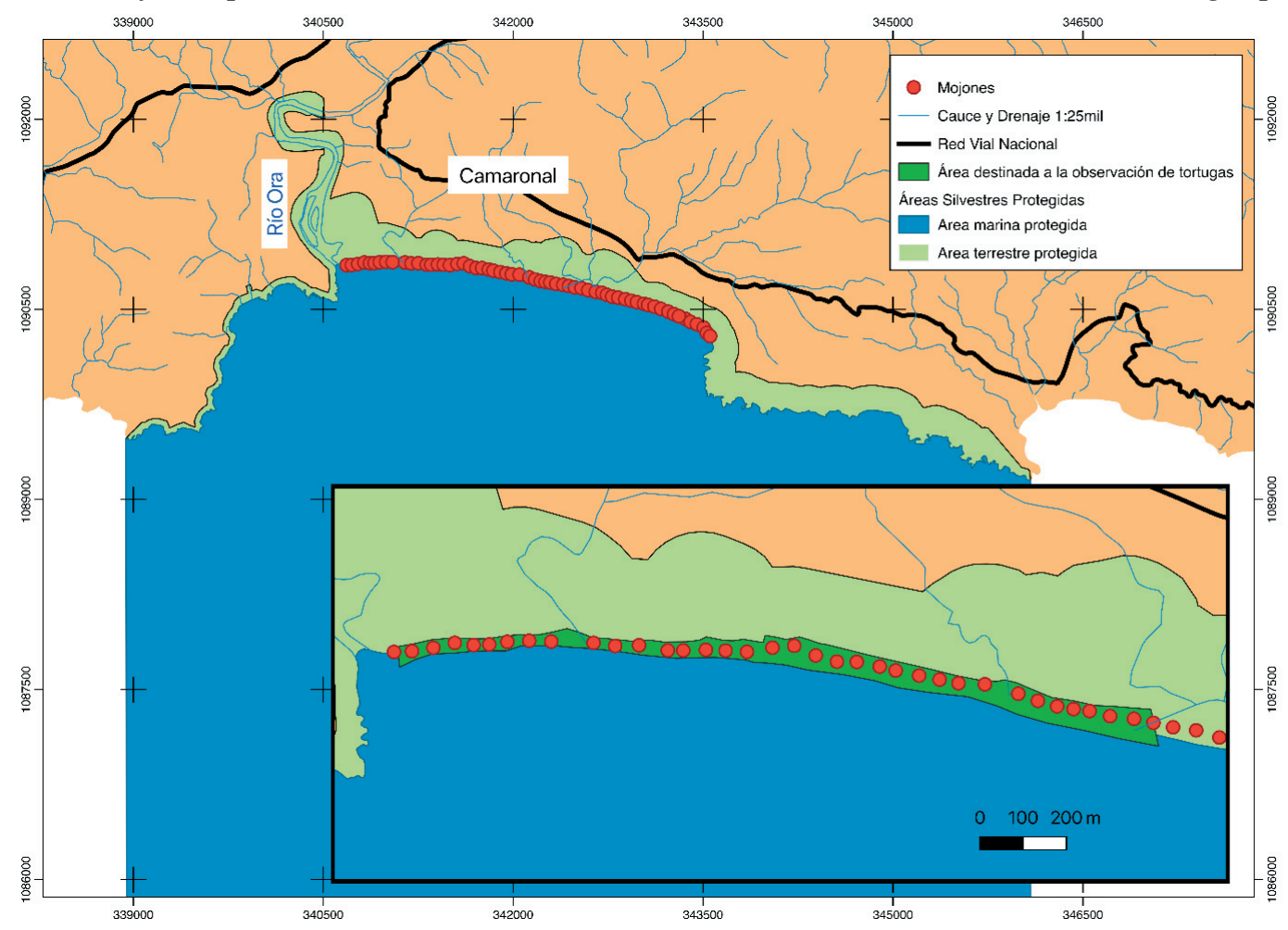

Figura 1. Zonificación del Refugio de Vida Silvestre Camaronal para la observación de tortugas marinas. Proyección CRTM05 and Datum WGS84.

Figure 1. Zones classification of the Camaronal Wildlife Management Refuge, Costa Rica, to observe marine turtles. CRTM05 Projection and WGS84 Datum. 
empresarios turísticos de las comunidades de Samara, Nosara, Carrillo, Estrada y Punta Islita. Estos empresarios aprovechan la anidación frecuente de L. olivacea en playa Camaronal.

El recorrido turístico se realiza con un tour operador en horas de la noche y según la marea; se prefiere que la marea sea de media a creciente. En compañía de un guía local y diferentes guías de los tours operadores, en conjunto con el grupo de turistas (número variable), bajan a la playa y proceden a caminar con linternas con filtros de luz roja. Dependiendo del grupo, los turistas pueden esperar en playa o bien caminar junto con los guías. Una vez que un guía identifica una tortuga, procede a coordinar para llamar al grupo de turistas a presenciar las diferentes etapas del proceso de anidación de la tortuga (i.e., las fases de preparar el sitio para la cámara de incubación, hacer el hueco, poner los huevos, tapar, camuflar el nido y retornar al agua). La mayoría de las veces, los empresarios turísticos prefieren ver cada uno una tortuga diferente por separado; sin embargo, hay noches en las que por la condición lunar, la época del año y la marea resulta muy difícil observar más de una tortuga y, potencialmente, muchos grupos de turistas pueden presenciar la misma tortuga anidando.

\section{Población monitoreada}

Del ensamble de tortugas marinas que anidan en el RNVSC, la mayor anidación corresponde a la tortuga lora, con un $99 \%$ de los eventos de anidación registrados, por lo que nos limitamos a analizar esta especie. Se incluyeron, además, datos de monitoreo de las temporadas de anidación 2013 y 2014 para estimar la abundancia de arribos. Generalmente, L. olivacea mantiene el comportamiento de anidación durante todo el año, con picos durante la estación lluviosa (Hart et al. 2014). La abundancia de arribos observados por día se relacionó con las fases lunares mediante los protocolos propuestos en el Manual para Mejores Prácticas de Conservación de las Tortugas Marinas en Centroamérica (Chacón et al. 2000), por su importancia para el proceso de anidación (Law et al. 2010).

\section{Cálculo del área de observación de tortugas marinas}

La estimación del área de observación de tortugas marinas (AOT) se obtuvo mediante una ecuación matemática desarrollada para los fines de este estudio. Esta ecuación busca regular el avistamiento de tortugas marinas en áreas protegidas, con base a variables de comportamiento de la especie, atributos espaciales (distribución de los objetos en el terreno) y relacionadas con el manejo de grupos de turistas. La ecuación fue formulada a partir de la distribución de las distancias entre tortugas en las playas en fase de anidación, la distancia recomendada entre un observador y la tortuga y el espacio mínimo vital que requiere una persona. La AOT genera un área circular, dentro de la cual un grupo limitado de turistas acompañados de un guía oficial se colocan para presenciar el proceso de desove de una tortuga marina de forma ordenada y sin afectar el comportamiento natural de la especie L. olivacea. Por tanto, se propone la siguiente expresión matemática para estimar la AOT:

$$
A O T=\left(\frac{D T}{2}+D I+D E P\right)^{2} \pi \quad \text { Ecuación } 1
$$

En la Figura 2 se ilustra la operatividad de las distancias y áreas que se aplican para la observación de tortugas marinas por grupos de turistas. La AOT debe ser aplicada después de tener clara la zonificación del área protegida, en la que se recomienda delimitar el área total de anidación (AE) y un área exclusiva de anidación (AEX). En particular, la AOT debe aplicarse a una zona predefinida denominada Zona de Observación de Tortugas Marinas (ZOM). La ZOM consiste en el área utilizada para que los visitantes del ASP puedan disfrutar de la observación de tortugas marinas. La ZOM se obtiene mediante la Ecuación 1.

$$
Z O M=A E-A E X
$$

Ecuación 2

Área total anidación (AE). La AE es el área utilizada por L. olivacea para realizar el proceso de anidación.

Área exclusiva de anidación (AEX). La AEX es el área excluida de la visitación turística por las condiciones de fragilidad ecológica que presenta. Por ejemplo, playas arenosas contiguas a esteros, manglares, ríos, entre otros.

Distancia entre grupos de observadores de una tortuga marina (DT). Es la distancia promedio entre en grupo y otro de observadores de un individuo de tortuga marina que se encuentra realizando su proceso 


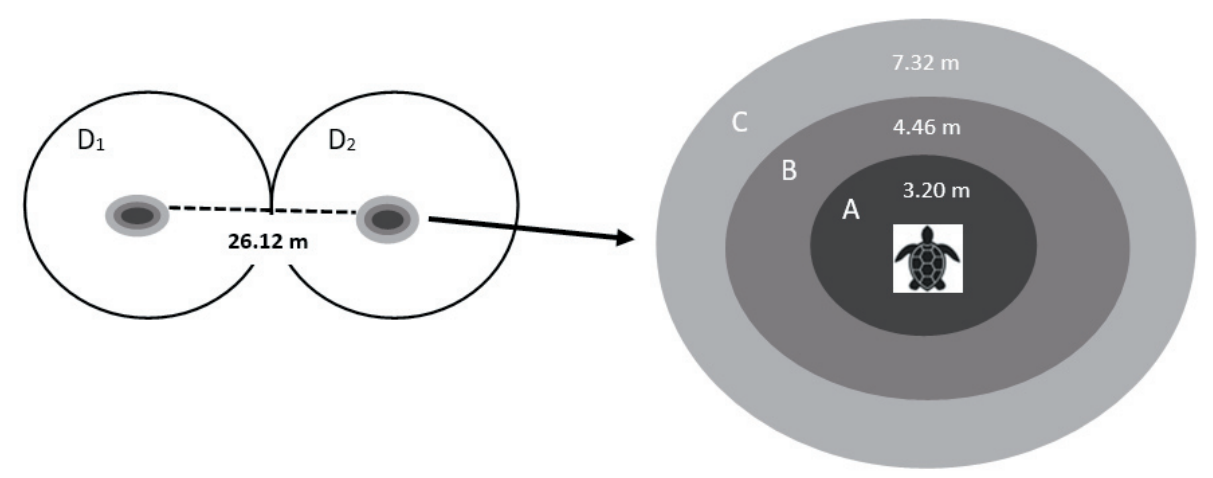

Figura 2. Diagrama operativo para la aplicación de la ecuación del AOT en áreas silvestres protegidas marino-costeras. A: área que requiere la tortuga marina para realizar su proceso de anidación; B y C: área que ocupan los observadores de tal forma que se mantenga un área mínima de $5 \mathrm{~m}^{2}$ por observador; D: área de observación de una tortuga marina por un grupo de turistas.

Figure 2. Operative diagram to apply the AOT equation in costal-marine protected areas. A: area required by sea turtles for their nesting process; $B$ and C: area occupied by observers in such a way that a minimum area of $5 \mathrm{~m}^{2}$ per observer is maintained; D: sea turtle viewing area by a group of tourists.

de anidación en la playa. El promedio de esta distancia, así como su intervalo de confianza al 95\%, se determinó con datos de arribos en octubre 2015. Además, se identificaron la luna y la marea más favorables para la ocurrencia de arribos en la playa de anidación, las cuales corresponden con la fase menguante y la marea media entre la pleamar y baja mar. Se midió la distancia entre tortugas que se encontraban simultáneamente en la playa bajo estas condiciones, para un total de 25 repeticiones $(n=25)$. Para ser seleccionados como repetición válida, se tenía como requisito que los individuos evaluados ingresaran a la playa de anidación al mismo tiempo a realizar el proceso de desove. Se utilizó el límite superior de la distancia entre tortugas marinas (DT) como radio para el cálculo del AOT.

Distancia entre grupos de turistas y una tortuga marina anidando (DI). Es la distancia que se designa entre el grupo de observadores y el individuo en actividad de anidación, por consenso entre sesiones de trabajo entre la administración del RNVSC y tour operadores de la zona de influencia al área protegida en donde se designó una distancia de $1.60 \mathrm{~m}$, que permite observar el proceso de ovoposición completo en el nido sin afectarlo. El largo del caparazón de L. olivacea es $60 \mathrm{~cm}$ y el intervalo de confianza al $95 \%$ para los individuos de mayor longitud fue 62.5-64.4 cm (Zug et al. 2006). Además, al valor de la media se le debe añadir una distancia de $1 \mathrm{~m}$ de movimiento del individuo durante el proceso de desove, que satisface el área de movimiento para individuos. Este valor se asume a partir de los valores registrados por Brenes et at. (2014) para el largo curvo del caparazón $(57-79 \mathrm{~cm})$ y para el ancho curvo del caparazón de $(62-79 \mathrm{~cm})$, los cuales están por debajo de $1 \mathrm{~m}$, permitiendo movimientos de la tortuga sobre su propio eje durante el proceso de anidación.

Distancia entre personas (DEP). Es el área mínima de la cual debe disponer una persona para disfrutar la actividad turística. Zumbardo (2017) recomienda un mínimo de $5 \mathrm{~m}^{2}$ por persona para un disfrute confortable. Por tanto, se designó un valor de $1.26 \mathrm{~m}$ como el radio mínimo para estimar el EP.

\section{El número de observadores (M)}

Adicionalmente a la estimación de la AOT se realizó una evaluación del tamaño de grupos de observadores de la actividad de anidación. Este valor corresponde al número de visitantes que pueden observar una tortuga sin afectar significativamente su tiempo de anidación, según el comportamiento propio de la especie. Para determinar el tamaño del grupo se adaptó la metodología utilizada para para C. caretta en California USA (Johnson et al. 1996). Esta metodología también requiere estimar el tiempo de anidación, es decir la duración de cada una de las etapas de la anidación. Esta estimación fue realizada a individuos que arribaron al refugio entre junio a diciembre del 2015. El tiempo de cada fase de anidación de la especie L. olivacea se realizó en presencia de grupos de turistas de 30, 25, 20, 15, 10 y 5 personas. Las fases consideradas fueron; tiempo en que la tortuga emerge, hace cama, realiza el hueco, desova, tapa, camufla y regresa al mar. Se utilizó un cronómetro y una hoja impresa con los datos pertinentes para el observador. Se tomaron otros datos como 
el tamaño del caparazón, sitio de anidación y presencia de heridas. Fueron realizadas 15 repeticiones de cada tipo de grupo y se registró el tiempo de anidación en cada fase y luego se obtuvo el promedio de duración.

Se ajusto un modelo linear generalizado (GLM) para relacionar el tipo de grupo de observadores, definido por la cantidad personas, y el tiempo que demoraba una tortuga para realizar el proceso de anidación con todas sus fases como variable respuesta. Se realizó el proceso de simulación de 100 muestras a partir del promedio aritmético y su desviación estándar asociada obtenida para cada grupo de turistas utilizando la herramienta random de la librería Pandas para el lenguaje Python (Python Software Foundation, Python Language Reference, versión 2.7). Con el conjunto de datos obtenido a partir de la simulación se tomó una muestra del 30\% de los datos para entrenar el modelo y finalmente se obtuvieron los valores predictivos con el $70 \%$ de los datos. Este análisis nos permitió definir cuál es tamaño de grupo de observadores es óptimo para no afectar el comportamiento de anidación de la especie. Por ejemplo, Hughes and Richard (1974) reportaron una duración total de la anidación en 45 minutos, mientras que Vega and Robles (2004) reportaron una media de 44.6 minutos (34-66.7 $\mathrm{min}$ ) sin presencia de turistas. La fase en que se encontró la tortuga en el momento del avistamiento no fue tomada en cuenta para análisis, ya que no se sabe cuánto tiempo lleva en esa categoría de comportamiento. En función a los registros de anidación del RNVSC para los años 2013, 2014 y 2015, en donde la cantidad sea menor a 10 tortugas marinas para una noche en específico, no se realiza la observación de tortugas marinas y esta tendencia se realiza en los meses secos del año, entre enero y abril, respectivamente.

\section{Resultados}

\section{Anidación de L. olivacea en el RNVSC}

Durantela temporada 2013 fueron registrados 2429 arribos, para 2014 se registraron 6323 y en 2015 se reportaron 6763. Los arribos ocurrieron con mayor frecuencia durante el período lluvioso, mientras que durante el período seco se reportaron meses con menos de 10 arribos (Figura 3). Además, bajo influencia de la luna menguante se dieron la mayor cantidad de arribos en contraposición con la luna nueva (esto, para ambos períodos climáticos) (Figura

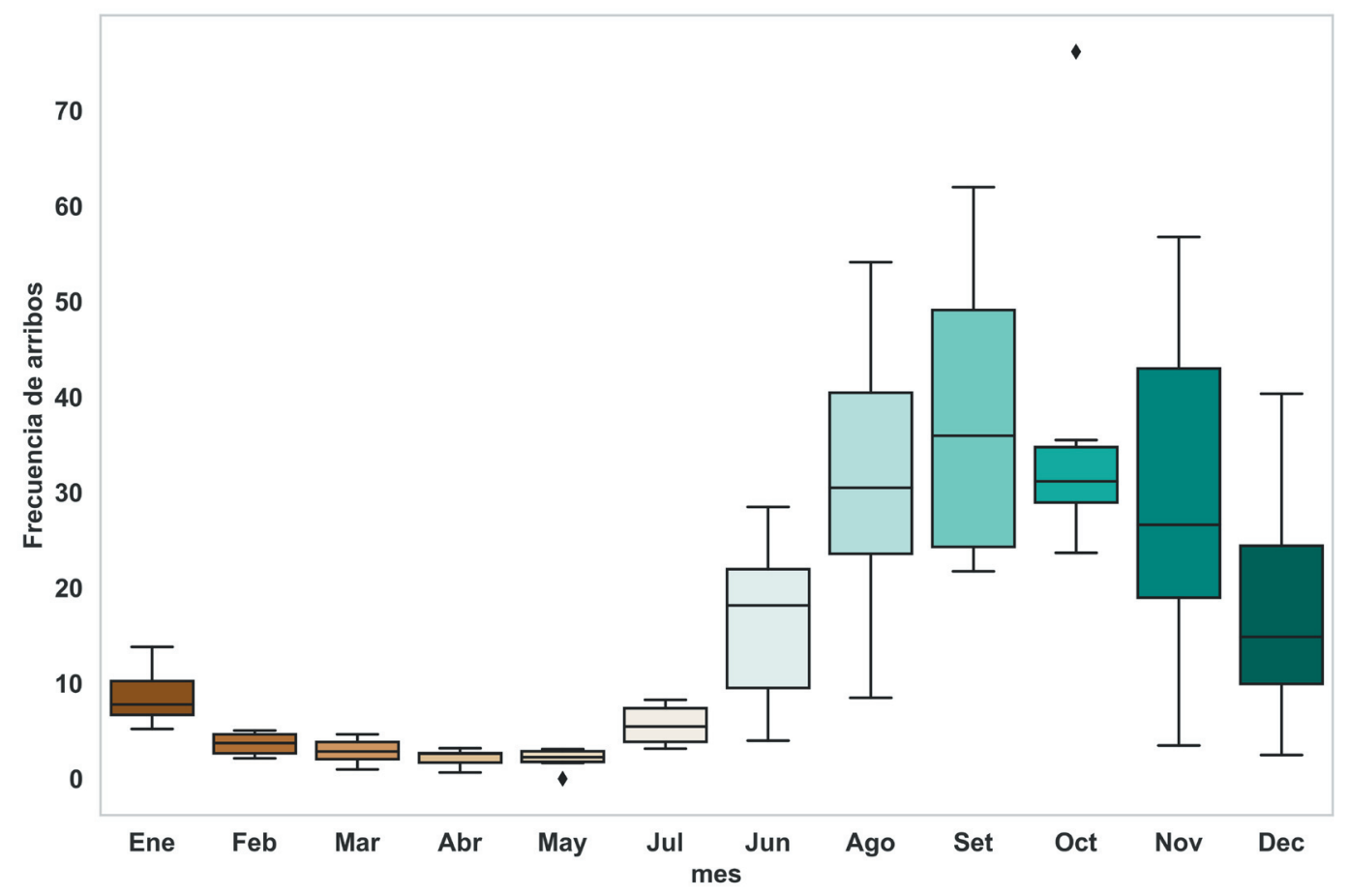

Figura 3. Frecuencia de arribos totales mensuales de la especie L. olivacea registrados entre 2013 y 2015 en el Refugio de Vida Silvestre Camaronal, Costa Rica.

Figure 3. Monthly frequency of olive turtle arrivals L. olivacea recorded between 2013 and 2015 in Camaronal Wildlife Management Refuge, Costa Rica. 
4). Cabe destacar que durante el período de febrero a marzo en todas las temporadas evaluadas pueden presentarse días con menos de 10 arribos de tortugas marinas.
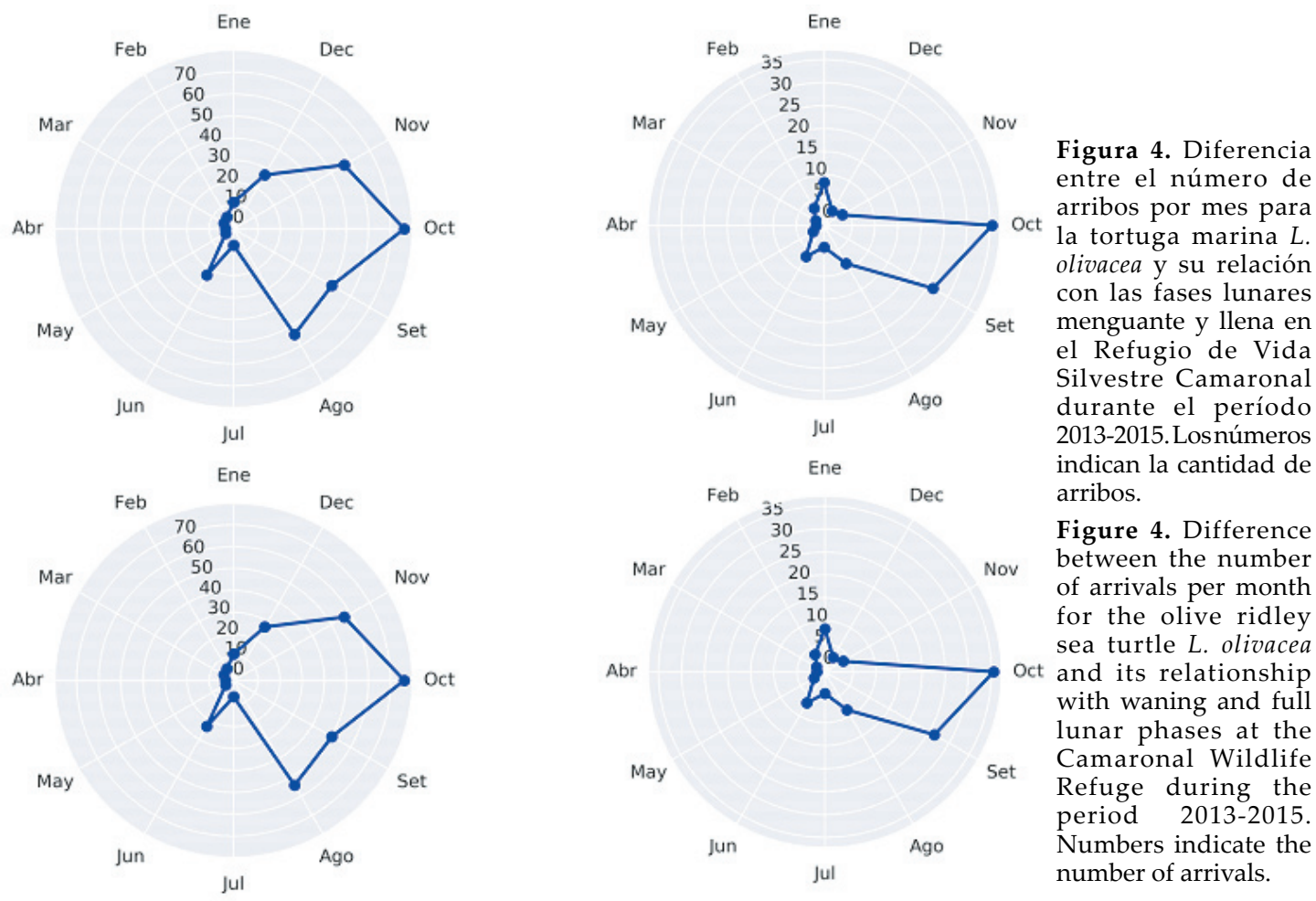

Figure 4. Difference between the number of arrivals per month for the olive ridley sea turtle L. olivacea oct and its relationship with waning and full lunar phases at the Camaronal Wildlife Refuge during the period 2013-2015. Numbers indicate the number of arrivals.

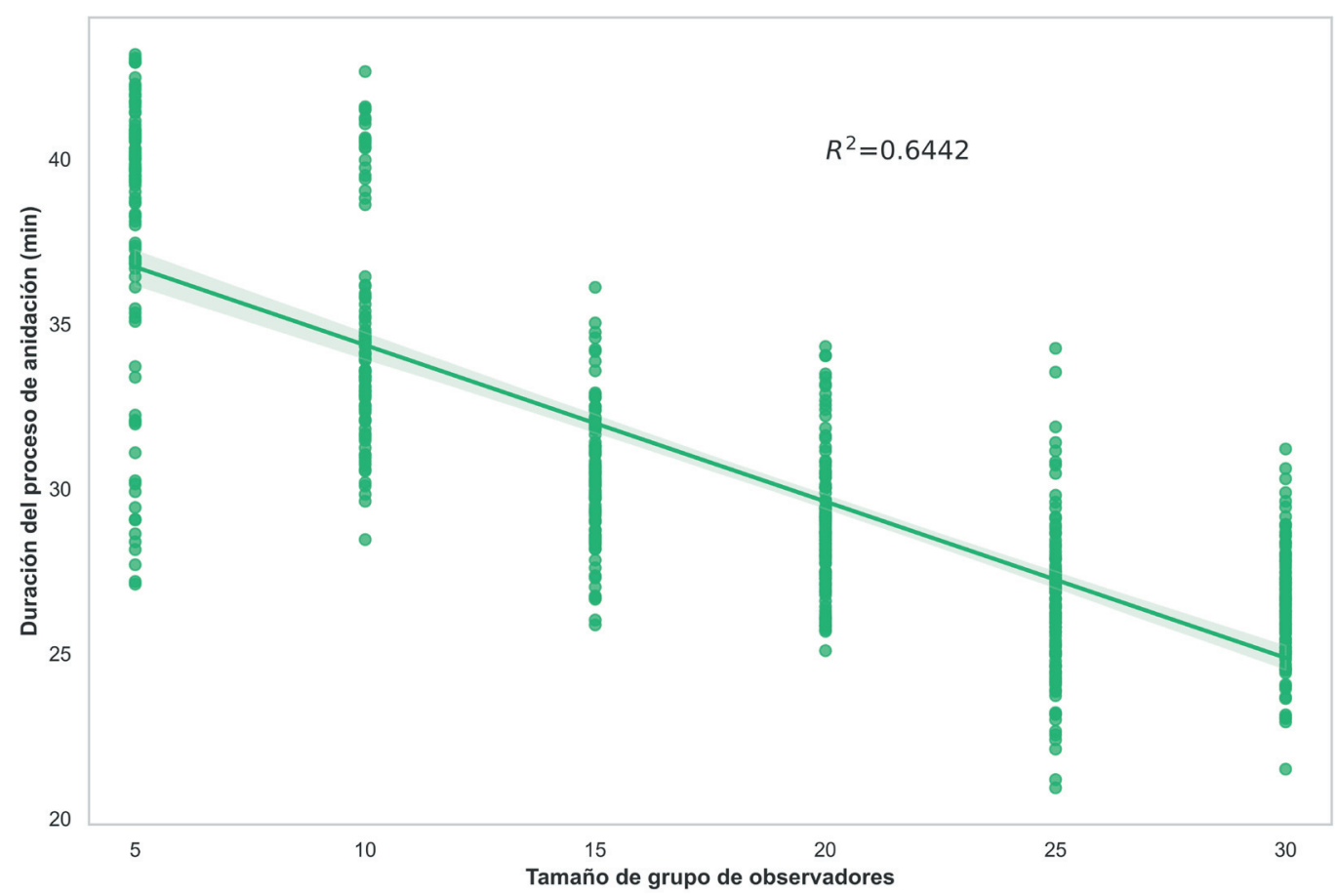

Figura 5. Modelo lineal generalizado entre los tipos de grupos de observadores y el tiempo (en minutos) utilizado por las tortugas marinas (L. olivacea) para completar las fases de anidación en el Refugio de Vida Silvestre Camaronal, Costa Rica.

Figure 5. General linear model between observers group types and the time (in minutes) required by sea turtles ( $L$. olivacea) to complete nesting phases in the Camaronal Wildlife Refuge, Costa Rica. 


\section{Cálculo de la AOT}

Los valores para las diferentes categorías de área se describen en la Figura 2. El modelo lineal generalizado permitió establecer una relación entre número de observadores y el tamaño de los grupos $\left(R^{2}=0.64, \mathrm{MSE}=9.03\right.$, $\mathrm{DS}=0.0365, P<0.001)$, y que a mayor cantidad de observadores disminuye el tiempo total utilizado por la tortuga marina para completar las fases de anidación (Figura 5). Para el cálculo del AOT se utilizó el valor de 10 observadores por ser el valor mínimo sin afectación (Figura 5), apegándose al principio precautorio. Aplicando la ecuación para el cálculo del AOT con los valores de las variables (Tabla 1) obtenemos lo siguiente:

$A O T=(9.74 m+1.60 m+1.216 m)^{2} \pi \quad$ Ecuación 3

El valor del AOT fue $499.18 \mathrm{~m}^{2}$, lo que en términos prácticos y operativos para la gestión del turismo de observación de tortugas marinas se puede redondearse a $500 \mathrm{~m}^{2}$.

Tabla 1. Resultados de las variables evaluadas para el cálculo del área de observación de tortugas marinas en el Refugio de Vida Silvestre Camaronal, Costa Rica.

Table 1. Results of the variables evaluated for the calculation of the sea turtle observation area in the Camaronal Wildlife Refuge, Costa Rica.

\begin{tabular}{lcc}
\hline Variable & Unidad & Valor \\
\hline $\begin{array}{l}\text { Zona de observación de } \\
\text { tortugas marinas (ZOM) }\end{array}$ & $\mathrm{m}^{2}$ & 514000 \\
$\begin{array}{l}\text { Área total de anidación } \\
\text { (AE) }\end{array}$ & $\mathrm{m}^{2}$ & 85585 \\
$\begin{array}{l}\text { Área exclusiva de } \\
\text { anidación (AEX) }\end{array}$ & $\mathrm{m}^{2}$ & 34000 \\
$\begin{array}{l}\text { Distancia entre tortugas } \\
\text { marinas (DT) }\end{array}$ & $\mathrm{m}$ & $\begin{array}{c}14.75 \\
(19.48-10.03)\end{array}$ \\
\hline
\end{tabular}

\section{Discusión}

Las fases de anidación en tortugas marinas son claves para mantener la conservación de las especies, pues corresponden al proceso de producción de nuevos individuos. Cada fase en la nidificación cumple un objetivo para garantizar el éxito de eclosión de la camada; por ejemplo, el tiempo dedicado a la construcción del nido permite darle profundidad al mismo y generar varias capas de huevos, de los cuales aquellos que ocupen las capas inferiores tienen mayores posibilidades de eclosionar debido a que mantienen una temperatura y una humedad adecuada y son menos susceptibles a la depredación (Engeman et al. 2005) y la extracción (López and Mora 2012). Nuestra investigación sugiere que debe mantenerse un número precautorio de turistas observando el proceso de anidación para no aumentar los tiempos de cada fase y no afectar la dinámica poblacional de las tortugas marinas. Desde el punto de vista biológico, el tamaño de grupo que sugerimos $(n=10)$ no genera alteraciones significativas en el comportamiento de anidación de la tortuga loro (Johnson et al. 1996). Sólo en los grupos de entre 5 y 10 observadores se alcanzan los promedios reportados en otros estudios (45.0 y 44.6 minutos) (Vega and Robles 2004; Huges and Richard 1974); de ahí la importancia de definir como regla grupos de turistas iguales o menores a 10 personas. Las fases lunares con mayor cantidad de arribos coinciden con lo reportado por Plotkin et al. (1997), quienes señalan las mejores condiciones de marea para realizar su proceso de anidación (James and Melero 2014).

Las decisiones de manejo tienen un impacto directo sobre los objetos de conservación en las áreas protegidas, y por esto se espera que sean tomadas con base en evidencia técnica y rigurosa (Polasky et al. 2011; Chacon and Soley 2020). Sin embargo, la información necesaria para el manejo de los objetos de conservación a menudo es poca o no existe, y peor si se trata de áreas marinas protegidas (Lundquist and Granek 2005; Chacon and Soley 2020). El aporte de la información técnica lograda para la estimación de la AOT para el avistamiento de la tortuga lora en el RNVSC podrá ser replicada y adaptada en otras áreas protegidas en Costa Rica, que tienen como objeto de conservación a las tortugas marinas. A través del plan de manejo como instrumento de planificación de la gestión de las ASP y el plan específico de turismo, la AOT podría ser monitoreada a través de la aplicación de la guía de efectividad de la gestión de las ASP. Poder tener un manejo adaptativo con respecto a los resultados que se recolecten, y que permita actualizar la información, hará más robusta la herramienta de planificación generada en este trabajo para las ASP de Costa Rica que tengan las tortugas marinas como objetos de conservación.

Los tamaños de grupo de 30 y 25 observadores registraron perturbación en la anidación de L. olivacea, pudiendo ocasionar hasta la interrupción del proceso de anidación. Se han documentado cambios en el comportamiento de distintas especies durante actividades turísticas como caminatas, buceo apnea y buceo autónomo, pues asustan a los animales e, incluso, hacen que huyan 
de los sitios habituales, interrumpiendo o modificando comportamientos de su historia natural (e.g., ovoposición en tortugas marinas, cuidado parental en machos de osos polares, el patrullaje en machos de lobos marinos, entre otros [Labrada 2003; McClung et al. 2004]). Otro caso reportado es para la tortuga verde (C. mydas) en el Parque Nacional Galápagos, cuyos individuos huyen ante la proximidad de los botes, causando distanciamiento de sitios de forrajeo y, en general, alteración de su comportamiento (González and Cubero 2010). En Costa Rica, aunque históricamente siempre se han recomendado diez observadores para interactuar con la vida silvestre, a partir del reglamento de uso público del Refugio Nacional de Vida Silvestre Ostional y otras metodologías (MIRENEM 1994) no se había fundamentado técnicamente este valor u otro en el comportamiento real de la anidación de la tortuga lora. Con este estudio se logra fundamentar y recomendar el tamaño ideal de grupo de observadores.

Con respecto a la frecuencia de arribos de tortugas marinas, se aprecia cómo a lo largo del año existen meses favorables y desfavorables para la observación. Esto concuerda con el comportamiento de anidación de la especie L. olivacea, cuyo pico reproductivo coincide con la época de mayor precipitación (Romero et al. 2007). De acuerdo con los resultados (Figura 3), es posible distinguir claramente tres temporadas en el RNVSC: la primera es una temporada seca que abarca de diciembre a mayo, en la que no se recomienda la observación de tortugas marinas según el monitoreo de tortugas marinas de los años 2013-2015; la segunda, una temporada de transición entre la época seca y lluviosa (mayo a julio), en la que observar la especie resulta muy irregular; y finalmente, una temporada lluviosa (julio a agosto), caracterizada por una fuerte anidación (Maìrquez 1996; Romero et al. 2007; Viejobueno and Arauz 2015). Sin embargo, en la época de mayor anidación se pueden dar cambios en la cantidad de arribos que se relacionan con la fase lunar. Por ejemplo, durante la fase menguante ocurre una mayor anidación de tortugas marinas (Azanza 2009). Los meses de mayor visitación en la observación de tortugas marinas en el Refugio Camaronal son noviembre y diciembre, ya que representan el inicio de la temporada fuerte del sector turístico (ICT 2015) y coincide con una alta anidación de tortugas marinas en playa (SINAC 2014), lo que potencia el número de recorridos nocturnos en el área silvestre protegida.
Al aplicar la AOT se condiciona un límite máximo de visitantes que el sistema puede alcanzar para una noche puntual. Hinestroza y Páez (2000) recomiendan que en noches en que el recurso tortugas se encuentre disponible se debe estimar un número de turistas que represente el límite máximo que el sistema puede soportar, tomando en cuenta factores biológicos de la especie, temporales y de ubicación espacial de los grupos de observación. Es importante resaltar que la AOT requiere una zonificación previa de la playa de anidación para privilegiar sitios exclusivos para el arribo, pues la tortuga lora tiene la capacidad de detectar a través del olfato ciertas variables como temperatura, sedimentos, calidad de la arena y humedad. Esto les permite identificar cuáles sitios son favorables para la ovoposición y emergencia de sus crías (Bolongaro 2010). La zonificación permite reducir la compactación de la playa de anidación, facilitando el intercambio gaseoso en los nidos y la emergencia exitosa de los neonatos (lo que aumenta su potencial de mortalidad) (Eckert et al. 2000). La especie $L$. olivacea es más sensible a esta compactación por visitación al compararla con otras especies de tortugas marinas, ya que, por su anatomía y comportamiento, tienen la capacidad de colocar y elaborar la cámara de incubación a profundidades menores en comparación con otras especies marinas (Chacón et al. 2007).

El uso de la AOT es una herramienta de manejo que se suma al objetivo de regular las actividades turísticas que se realizan en playas de anidación. Esta eficiente herramienta permite gestionar correctamente el recurso a proteger, contemplando la dinámica del turismo (Huaraca 2008). Esto contribuye a su permanencia en el ecosistema, favorece la economía local y ayuda a cumplir los objetivos estratégicos del país para regular todas las acciones que se desarrollan en las áreas silvestre protegidas, especialmente en la conservación de los recursos. La tortuga marina L. olivacea constituye uno de los objetos de conservación del RNVSC y, por lo tanto, su preservación debe ser prioritaria (Dudley 2008).

Finalmente, resultará valioso que los resultados logrados sean socializados con todos los actores de la cadena de servicios ecosistémicos asociados al RNVC y que son beneficiados por el avistamiento de tortugas marinas, actividad que debe ser ordenada y regulada. Los actores incluyen guías locales, escuelas en el área de influencia del RNVSO 
y funcionarios, entre otros. Todos deben interiorizar la información técnico-científica lograda e incluirla en su discurso durante la atención de la visitación y enseñarle a los turistas los efectos negativos de sus conductas en la anidación de las tortugas marinas si no se respeta la AOT estimada, con su respectivo número de observadores.

Agradecimientos. Agradecemos al Ministerio del Ambiente y Energía a través del Área de Conservación Tempisque del Sistema
Nacional de Áreas Conservación por facilitar las instalaciones y logística para desarrollar esta investigación en el Refugio Nacional de Vida Silvestre Camaronal, Guanacaste, Costa Rica amparado en el permiso y resolución de Investigación ACT - OR - DR - 070 del año 2014. A los voluntarios que apoyaron la toma de datos durante el tiempo de estudio, funcionarios Agradecer al profesor Fernando Bermúdez y al Dr. Flavio Rodríguez por sus recomendaciones para la elaboración de este trabajo.

\section{REFERENCIAS}

Alió, J. J., and L. M. Altuve. 2010. Captura incidental y mortalidad de tortugas marinas en la pesquería industrial de camarones por arrastre en el noreste de Venezuela. Revista de Ciencias Marinas 36(2):161-178.

Alvarado, J., B. Herrera, L. Corrales, J. Asch, and P. Paaby. 2011. Identificación de las prioridades de conservación de la biodiversidad marina y costera en Costa Rica. Revista de Biología Tropical 59(2):829-842.

Amador, E., L. Cayot, M. Cifuentes, E. Cruz, and F. Cruz. 1996. Determinación de la capacidad de carga turística en los sitios de visita del Parque Nacional Galápagos. Servicio Parque Nacional Galápagos, Instituto Ecuatoriano Forestal y de Áreas Naturales y Vida Silvestre, Isla Galápagos, Ecuador.

Aranguren, J., J. Moncada, J. Neveda, D. Rivas, and C. Lugo. 2008. Evaluación de la capacidad de carga turística en la playa Canomita, Municipio Guanta, Estado Anzoátegui. Rev Invest 64:31-61.

Azanza, R. J., M. E. Ibarra, G. Gonzáles, F. A. Abreu, K. L. Eckert, G. Espinoza, and K. Oyama. 2013. Nesting ecology of Chelonia mydas (Testudines: Cheloniidae) on the Guanahacabibes Peninsula, Cuba. Revista de Biología Tropical 61(4):1935-1945. https://doi.org/10.15517/rbt.v61i4.12869.

Bickham, B., J. B. Inverson, J. Parham, H. D. Philippen, A. G. Philippen, A. G. Rhodin, H. B. Shaffer, and P. P. Dijk. 2007. An Annotated List of Modern Turtle Terminal Taxa with comments on Areas of Taxonomic Instability and Recent Change. Chelonia Research Monographs 4:173-199.

Brenes, O., J. Castro, V. Jiménez, A. Mora, and I. Mejia. 2004. Determinación de la capacidad de carga turística del Parque Internacional La Amistad. Centro Científico Tropical, San José, Costa Rica.

Brenes Arias, O., L. Bonilla Bonilla, A. Bonilla Salazar, and A. Vega Delgado. 2015. Características de la Anidación de Lepidochelys olivacea (Testudinata: Cheloniidae) entre el 2010 y 2012 en Playa Tortuga Ojochal de Osa, Puntarenas, Costa Rica. Revista de Biología Tropical 63:339-349. https://doi.org/10.15517/rbt.v63i1.23113.

Bolongaro, C. R., A. Z. Márquez García, V. Torres Rodríguez, and A. García-Vicario. 2010. Vulnerabilidad de sitios de anidación de tortugas marinas por efectos de erosión costera en el estado de Campeche. Pp. 73-96 en A. V. Botello, S. Villanueva Fragoso, J. Gutiérrez and J. L. Rojas Galaviz (eds.). Vulnerabilidad de las zonas costeras mexicanas ante el cambio climático. Universidad Autónoma de Campeche. Campeche, México.

Camacho, M. L., D. Amorocho, L. L. Mejía, M. J. Palacios, and G. F. Rondón. 2008. Caracterización genética de la colonia reproductiva de la tortuga marina golfina Lepidochelys olivacea en el Parque Nacional Natural Gorgona (Pacífico Colombiano) a partir de secuencias de ADN mitocondrial. Boletín de Investigaciones Marinas y Costeras 37(1):77-92.

Cifuentes, M. 1992. Determinación de la capacidad de carga en áreas protegidas. Serie técnica, informe técnico 194. Centro Agronómico de Investigación y Enseñanza, Turrialba, Costa Rica.

Cortés, J., and C. E. Jiménez. 2003. Corals and coral reefs of the Pacific of Costa Rica: history, research, and status. Pp. 361-385 en J. Cortés (ed.). Latin American Coral Reefs. Elsevier Science. Holanda. https://doi.org/10.1016/B978044451388-5/50017-5.

Chacón, I. S., and F. G. Soley. 2020. Investigación para la toma de decisiones de manejo en áreas marinas protegidas como la Isla del Coco, Costa Rica. Revista de Biología Tropical 68(supl. 1):S1-S17. https://doi.org/10.15517/ rbt.v68iS1.41126.

Chacón, D., J. Sánchez, J. J. Calvo, and J. Ash. 2007. Manual para el manejo y la conservación de las tortugas marinas en Costa Rica; con énfasis en la operación de proyectos en playa y viveros. Sistema Nacional de Áreas de Conservación (SINAC), Ministerio de Ambiente y Energía (MINAE). Gobierno de Costa Rica. San José. Pp. 103.

Dudley, N., and A. Phillips. 2006. Forests and Protected Areas: Guidance on the use of the UICN protected area management categories. UICN, Gland, Suiza y Cambridge, UK. https://doi.org/10.2305/IUCN.CH.2006.PAG.12.en.

Dudley, N. 2008. Directrices para la aplicación de las categorías de gestión de áreas protegidas. Gland, Suiza: UICN. https://doi.org/10.2305/IUCN.CH.2008.PAPS.2.es.

Eckert, K. L., K. A. Bjorndal, F. A. Abreu-Grobois, and M. Donnelly (eds.). 2008. Técnicas de Investigación y Manejo para la Conservación de las Tortugas Marinas. Grupo Especialista en Tortugas Marinas UICN/CSE Publicación No. 4. Pennsylvania, USA.

Engeman, R. M., R. E. Martin, H. T. Smith, J. Woolard, C. K. Crady, S. A. Shwiff, B. Constantin, M. Stahl, and J 
Griner. 2005. Dramatic reduction in predation on marine turtle nests through improved predator monitoring and management. Oryx 39(3):318-326. https://doi.org/10.1017/S0030605305000876.

Gámez, V., M. García, S. Osorio, G. Vázquez, and C. Constantino. 2009. Patología de las tortugas marinas (Lepidochelys olivacea) que arribaron a las playas de Cuyutlán, Colima, México. Veterinaria México 40(1):69-78.

Garay, G., G. Guineo, E. Mutschke, and C. Ríos. 2008. Tamaño, estructura y distribución estacional de poblaciones de aves acuáticas en el fiordo última esperanza y canal señor, región de Magallanes. Anales del Instituto de la Patagonia 36(2):33-44. https://doi.org/10.4067/S0718-686X2008000200004.

González, P. F., and P. P. Cubero. 2010. Efecto de actividades turísticas sobre el comportamiento de fauna representativa de las Islas Galápagos, Ecuador. Latin American Journal of Aquatic Research 38(3):493-500.

Hinestroza, L., and V. P. Páez. 2000. Anidación y manejo de la tortuga golfina (Lepidochelys olivacea) en la playa la cuevita bahía Solano. Chocó, Colombia. Cuadernos de Herpetología 14(2):131-144.

Huaraca, L. E. 2008. Monitoreo de impactos del turismo en la zona alta de la Reserva Ecológica Cayambe Coca. Tesis de maestría en Gestión Turística. Universidad Tecnológica Equinoccial, Ecuador. Pp. 110.

Hughes, D. A., and J. D. Richard. 1974. The nesting of Pacific Ridley Turtle Lepidochelys olivacea on Playa Nancite, Costa Rica. Marine Biology 24:97-107. https://doi.org/10.1007/BF00389343.

Instituto Meteorológico Nacional (IMN). 2015. Datos de precipitación de los últimos tres años de la estación en Barco Quebrado, Sámara, Guanacaste. Costa Rica.

James, R., and D. Melero. 2012. Anidación y conservación de la tortuga lora (Lepidochelys olivacea) en playa Drake, península de Osa, Costa Rica (2006 a 2012). Rev Biol Trop 63:117-129. https://doi.org/10.15517/rbt.v63i1.23099.

Johnson, S. A., K. A. Bjorndal, and A. B. Bolten. 1996. Effects of organized turtle watches on Loggerhead (Caretta caretta) nesting behavior and hatchling production in Florida. Conservation Biology 10(2):570-577. https://doi.org/10.1046/ j.1523-1739.1996.10020570.x.

Labrada, V. 2003. Influencia del turismo sobre la conducta del lobo marino de California Zalophus californianus en la lobería "Los Islotes", México. Tesis de Maestría en Ciencias, con Especialidad en Recursos Marinos. Universidad Autónoma de Baja California, Baja California, México. Pp 103.

Law, A., T. Clovis, G. R. Lalsingh, and J. R. Downie. 2010. The influence of lunar, tidal and nocturnal phases on the nesting activity of leatherbacks (Dermochelys coriacea) in Tobago, West Indies. Marine Turtle Newsletter 127:12-17.

López, I., and J. M. Mora. 2012. Vacíos para la Explotación Legal de Huevos de la Tortuga Lora (Lepidochelys olivacea) en el Refugio Nacional de Vida Silvestre Ostional, Costa Rica. Ceiba 53(2):95-108. https://doi.org/10.5377/ ceiba.v53i2.2507.

Lundquist, C. J., and E. F. Granek. 2005. Strategies for successful marine conservation: integrating socioeconomic, political, and scientific factors. Conservation Biology 19:1771-1778. https://doi.org/10.1111/j.1523-1739.2005.00279.x.

Hart, C., C. Ley-Quiñonez, A. Maldonado-Gasca, A. Zavala-Norzagaray, and A. Abreu-Grobois. 2014. Nesting characteristics of olive ridley turtles (Lepidochelys olivacea) on el Naranjo Beach, Nayarit, Mexico. Herpetological Conservation and Biology 9(2):524-534.

Márquez, R. 1996. Las tortugas marinas y nuestro tiempo. Fondo de cultura económica, México.

Ministerio de Recursos Naturales, Energía y Minas MIRENEM. 1994. Decreto N. ${ }^{\circ}$ 23150-Mirenem. Creación del Refugio Nacional de Vida Silvestre Camaronal Mixto. La Gaceta, 83. San José, Costa Rica.

McClung, M., P. Seddon, M. Massacre, and A. Setiawan. 2004. Nature-based impacts on yellow-eyed penguins. Biological Conservation 119(1):279-285. https://doi.org/10.1016/j.biocon.2003.11.012.

O' Reilly, A. M. 1986. Tourism carrying capacity: Concept and issues. Tourism Management 7(4):254-258. https: //doi.org/10.1016/0261-5177(86)90035-X.

Polasky, S., S. R. Carpenter, C. Folke, and B. Keeler. 2011. Decision making under great uncertainty: environmental management in an era of global change. Trends in Ecology and Evolution 26:398-404. https://doi.org/10.1016/ j.tree.2011.04.007.

Plotkin, P. T., D. C. Rostal, R. A. Byles, and D. W. Owens. 1997. Reproductive and Developmental Synchrony in Female Lepidochelys olivacea. Journal of Herpetology 31(1):17-22. https://doi.org/10.2307/1565323.

Pullin, A. S., T. M. Knight, D. A. Stone, and K. Charman. 2004. Do conservation managers use scientific evidence to support their decision-making? Biological Conservation 119:245-252. https://doi.org/10.1016/j.biocon.2003.11.007.

Romero, G., L. M. Hernández, B. García, A. Santos, and O. Meyer. 2007. Protección y conservación de tortugas marinas de la zona costera de Michoacán, México. Avances en Investigación Agropecuaria 11:2-9.

Rojas, N. 2011. Cuenca ríos Península de Nicoya. Ministerio de Ambiente y Energía, Costa Rica. San José, Costa Rica.

Rosales, C. A., M. Vera, and J. Llanos. 2010. Varamientos y captura incidental de tortugas marinas en el litoral de Tumbes, Perú. Revista Peruana de Biología 17(3):293-301. https://doi.org/10.15381/rpb.v17i3.4.

Sistema Nacional de Áreas de Conservación (SINAC). 2014. Plan de Manejo del Refugio Nacional de Vida Silvestre Camaronal, 2007-2012. Área de Conservación Tempisque. Nicoya, Guanacaste.

Sistema Nacional de Áreas de Conservación SINAC. 2016. Reglamento de uso público del Refugio Nacional de Vida Silvestre Camaronal, Resolución administrativa ACT-OR- DR- 052-16. San José, Costa Rica.

Stankey, G. H., D. N. Cole, R. C. Lucas, M. E. Petersen, and S. S. Frissell. 1985.The limits of acceptable change (LAC). System for Wilderness Planning. USDA Forest Service Intermountain Research Station. Ogden. Utah. https://doi.org/ 10.5962/bhl.title.109310

Vega, A. J., and Y. Robles. 2005. Descripción del proceso de anidación y biometría de hembras, huevos y nidos de tortuga 
golfina Lepidochelys olivacea (Eschscholtz, 1829), en Isla de Cañas, Pacífico Panameño. Tecnociencia 7(2):43-55.

Viejobueno, S., and R. Arauz. 2015. Conservación y actividad reproductiva de tortuga lora (Lepidochelys olivacea) en la playa de anidación solitaria en Punta Banco, Pacífico Sur de Costa Rica. Recomendaciones de manejo a través de dieciséis años de monitoreo. Revista de Biología Tropical 63:383-394. https://doi.org/10.15517/rbt.v63i1.23117.

Zug, G. R., M. Chaloupka, and G. H. Balazs. 2006. Age and growth in olive ridley seaturtles (Lepidochelys olivacea) from the North-central Pacific: a skeletochronological analysis. Marine Ecology 27:263-270. https://doi.org/10.1111/j.14390485.2006.00109.x.

Zumbardo, F. 2017. Manejo de visitantes y atención del turista en áreas protegidas costeras. Estudio de capacidad de carga en el parque nacional Marino Ballena, Costa Rica. Revista Interamericana de medioambiente y turismo 13(1): 69-90. https://doi.org/10.4067/S0718-235X2017000100068. 